\title{
The pion-pion scattering lengths from DIRAC
}

\author{
C. Santamarina \\ Basel University, Switzerland \\ on behalf of DIRAC Collaboration
}

\begin{abstract}
The scattering lengths of a two pion system are the golden magnitudes to test the QCD predictions in the low energy sector. The DIRAC (PS-212) experiment at CERN will obtain a particular combination of the S-wave isospin 0 and 2 scattering lengths by measuring the lifetime of pionium, the hydrogen-like $\pi^{+} \pi^{-}$atom. This measurement tests the accurate predictions of the Chiral Perturbation Theory. The most recent experimental results are presented.
\end{abstract}

\section{INTRODUCTION}

DIRAC experiment, conducted at CERN, is measuring the lifetime of the ground state of pionium, the hydrogen-like $\pi^{+} \pi^{-}$atom [1]. The decay of pionium is dominated by the strong channel $\pi^{+} \pi^{-} \rightarrow \pi^{0} \pi^{0}(\mathrm{BR}=94 \%)$. The ground state lifetime is related to the $\mathrm{S}$-wave isospin 0 and 2 scattering length difference by the Deser type formula known to NLO [2]:

$$
\frac{1}{\tau}=\frac{2}{9} \alpha^{3} \sqrt{M_{\pi}^{2}-M_{\pi^{0}}^{2}-\alpha^{2} M_{\pi}^{2} / 4}\left|a_{0}^{0}-a_{0}^{2}\right|^{2}(1+\delta),
$$

where $\alpha$ is the fine structure constant, $M_{\pi}$ the mass of the charged pions, $M_{\pi^{0}}$ the mass of the neutral pion and $\delta$ the correction to $\operatorname{NLO}(\delta=0.058)$.

The Chiral Perturbation Theory has a precise prediction to $\mathscr{O}\left(p^{6}\right)$ for this difference and hence for the lifetime of pionium [3]:

$$
\left|a_{0}^{0}-a_{0}^{2}\right|=0.265 \pm 0.004 \Rightarrow \tau=(2.9 \pm 0.1) \cdot 10^{-15} s
$$

which must be checked with similarly accurate experimental data. DIRAC goal is to achieve a $5 \%$ precision in the determination of the scattering length difference by measuring the lifetime with $10 \%$ precision.

\section{EXPERIMENTAL METHOD}

DIRAC measures the pionium lifetime analyzing the low relative momentum $(\vec{Q})$ spectrum of $\pi^{+} \pi^{-}$pairs produced in the collisions of the $24 \mathrm{GeV} / \mathrm{c}$ proton beam with the target. The target consists of one or several metal layers of the overall thickness of $\sim 100 \mu \mathrm{m}$. 

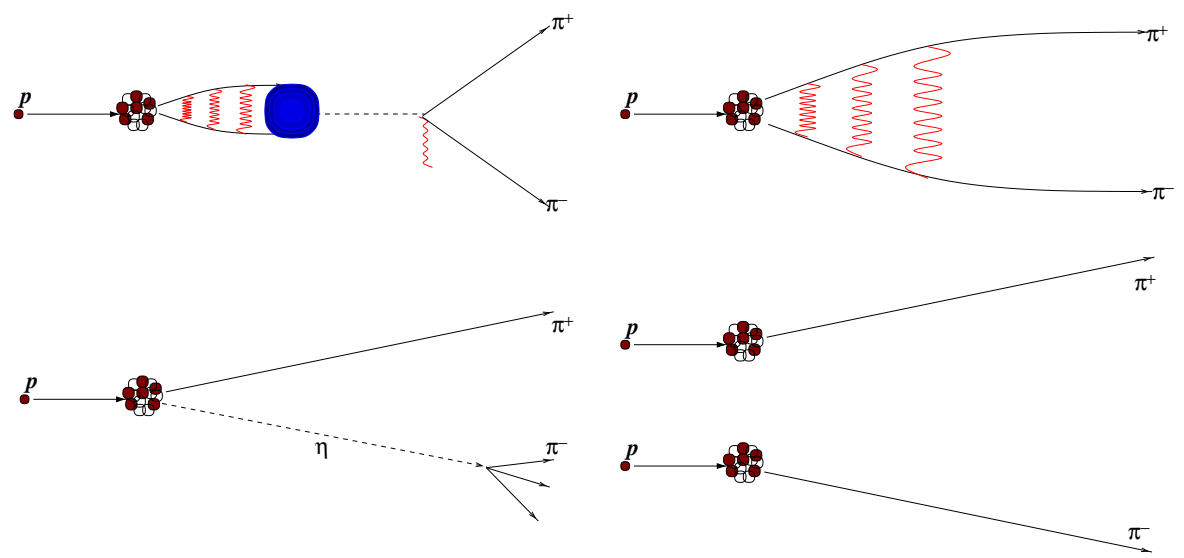

FIGURE 1. Pion pair production mechanisms: Top left:Atomic Pair. Top right:Coulomb Pair. Bottom left:Non Coulomb Pair. Bottom right: Accidental Pair.

This spectrum is formed of Real (time correlated) pairs, in which both pions come from a single proton-target interaction, and Accidentals, coming from two different interactions. Time correlated pairs are divided into:

- Atomic Pairs (pairs resulting from atomic break-ups). Pionic atoms are created by the Final State Coulomb Interaction (FSCI) of low relative momentum $\pi^{+} \pi^{-}$pairs. The sample of initial pionic atoms $\left(N^{A}\right)$ evolves by colliding with the atoms of the metal target. The evolution terminates when the atoms break-up in one of the collisions or annihilate. If the number of broken atoms is given by $n^{A}$ then, the shorter is the lifetime of pionium the smaller is the break-up probability $P_{b r}=$ $n^{A} / N^{A}$. The dependence of the break-up probability on the lifetime is accurately known [4], as shown in figure 3 for the Nickel target. Atomic Pairs are restricted to the $Q<4 \mathrm{MeV} / \mathrm{c}$ region.

- Coulomb Pairs. Their production mechanism is the same as for the atoms but leading to continuous spectrum eigenstates of the hydrogen-like hamiltonian of the $\pi^{+} \pi^{-}$system. This is why the yield of atoms is proportional to the number of Coulomb pairs within a determined $\vec{Q} \in \Omega$ arbitrary region ${ }^{1}[5]$ :

$$
N^{A}=K \times \int_{\vec{Q} \in \Omega} \frac{d N^{C}}{d Q} .
$$

The relative momentum distribution of Coulomb Pairs is given by:

$$
\frac{d N^{C}}{d Q} \propto \mathscr{T}\left[\frac{2 \pi M_{\pi} \alpha / Q}{1-e^{-2 \pi M_{\pi} \alpha / Q}} Q^{2}\right]
$$

where $\mathscr{T}$ stands for the transformation due to multiple scattering in the target and setup resolution of the at production distribution between brackets.

\footnotetext{
${ }^{1}$ In the experiment usually $\Omega=\{$ Events with reconstructed $Q<2 \mathrm{MeV} / \mathrm{c}\}$.
} 
- Non Coulomb Pairs. Non Correlated pairs in which one of the pions comes from the decay of a long-lived source ${ }^{2}$. Its relative momentum distribution is purely phase space driven and, hence, given by

$$
\frac{d N^{N C}}{d Q} \propto \mathscr{T}\left[Q^{2}\right]
$$

Apart from time correlated pairs also Accidental $\pi^{+} \pi^{-}$Pairs, where each pion comes from a different proton-target interaction, are recorded. Their relative momentum distribution is also determined by phase space:

$$
\frac{d N^{A c c}}{d Q} \propto \mathscr{T}\left[Q^{2}\right] .
$$

The Accidental Pairs sample is used to parameterize the Real Pairs pairs spectrum:

$$
\frac{d N^{\text {Real }}}{d Q}=\frac{d n^{A}}{d Q}+\frac{d N^{N C}}{d Q}+\frac{d N^{C}}{d Q}=\frac{d n^{A}}{d Q}+(a R(Q)+b) \frac{d N^{A c c}}{d Q}
$$

where $R(Q)$ is the ratio between Coulomb pairs and accidentals, obtained with Monte Carlo, and $a$ and $b$ are calculated by fitting the spectrum in the $Q>4 \mathrm{MeV} / \mathrm{c}$ region, free from atomic pairs.

The shape of the relative momentum distribution of Atomic Pairs can be obtained as:

$$
\frac{d n^{A}}{d Q}=\frac{d N^{\text {Real }}}{d Q}-(a R(Q)+b) \frac{d N^{A c c}}{d Q} .
$$

Moreover, the break-probability can then be experimentally determined:

$$
P_{b r}=\int \frac{d n^{A}}{d Q} / K \int_{\vec{Q} \in \Omega} a R(Q) \frac{d N^{A c c}}{d Q} .
$$

An alternative method consists of using pure Monte Carlo distributions for Coulomb, Non Coulomb and Atomic Pairs to parameterize the Real pairs spectrum:

$$
\frac{d N^{\text {Real }}}{d Q}=\alpha \frac{d n_{M C}^{A}}{d Q}+\beta \frac{d N_{M C}^{N C}}{d Q}+\gamma \frac{d N_{M C}^{C}}{d Q}
$$

$\alpha, \beta$ and $\gamma$ being parameters obtained from the fit to the whole spectrum [6].

\section{SPECTROMETER}

DIRAC spectrometer is a two arm telescope with a $1.65 T$ central magnet separating positive and negative charged particle channels [7]. It is located in the T8 channel of the

\footnotetext{
${ }^{2}$ A long lived source is a resonance with decay time large enough that it can fly a distance much larger than the pionium Bohr radius $(387 \mathrm{fm}$ ) from the primary interaction vertex.
} 


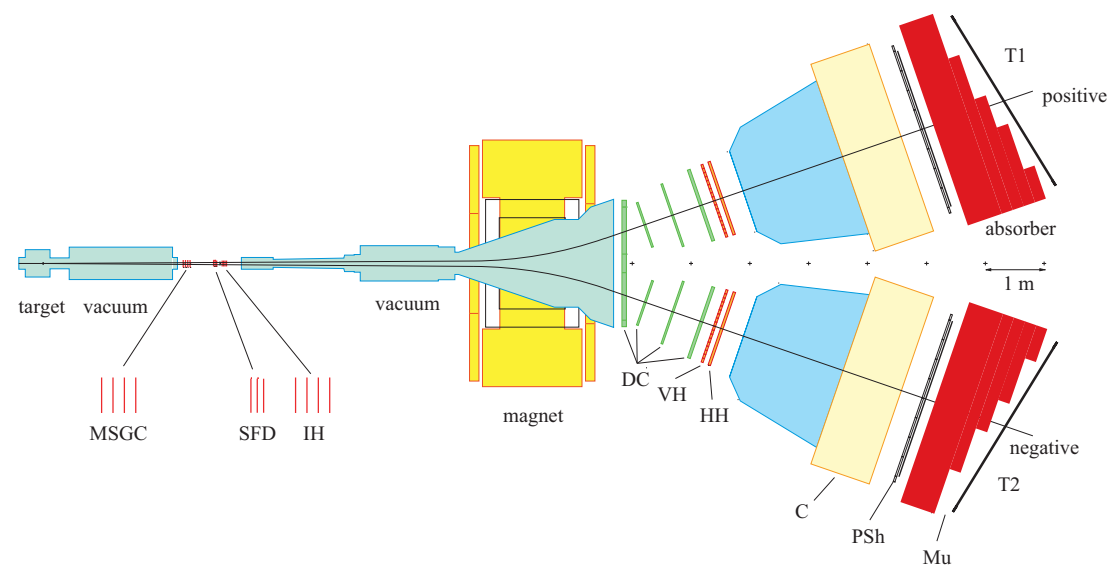

FIGURE 2. DIRAC spectrometer scheme. The top view shows the distribution of the detectors. MSGC=Micro Strip Gas Chambers, SFD=Scintillating Fiber Detector, IH=Ionization Hodoscopes detector, DC=Drift Chambers, VH=Vertical Hodoscopes, HH=Horizontal Hodospes, $\mathrm{C}=\mathrm{C}$ erenkov, $\mathrm{PSh}=$ Preshower, $\mathrm{Mu}=$ Muon counters.

PS East Hall B at CERN. The beam intensity is $\sim 10^{11} 24 \mathrm{GeV} / \mathrm{c}$ protons per spill $(0.4$ s).

The detectors are classified into upstream and downstream ones according to their position relative to the magnet. The upstream detectors consist of three sets of planes of Micro Strip Gas Chambers and Scintillating Fibers, used in tracking, and Ionization Hodoscopes, also used for triggering. Downstream we have two sets of Drift Chambers, the main tracking detector, Vertical and Horizontal hodoscope arrays for timing and triggering, Čerenkov detectors, to reject electrons at trigger level, Preshower, also used for triggering and electrons rejection offline, and muon detectors, for muons rejection.

The trigger system consists of three levels [8]. The T1 main trigger selects $\pi^{+} \pi^{-}$ events with coincidences upstream and the two arms downstream. The DNA+RNA makes a topological on-line analysis to perform the relative momentum cuts $Q_{x}<$ $3 \mathrm{MeV} / \mathrm{c}, Q_{y}<10 \mathrm{MeV} / \mathrm{c}$ and $Q_{l}<30 \mathrm{MeV} / \mathrm{c}$. Finally, the T4 level selects events containing one track per arm satisfying $Q_{x}<3 \mathrm{MeV} / \mathrm{c}$ and $Q_{l}<30 \mathrm{MeV} / c$ criteria.

The track resolution uses the standard Kalman filter procedure achieving an excellent relative momentum resolution of $\sigma_{Q_{x}}=\sigma_{Q_{y}}=0.4 \mathrm{MeV} / \mathrm{c}$ and $\sigma_{Q_{L}}=0.6 \mathrm{MeV} / \mathrm{c}$.

\section{EXPERIMENTAL RESULTS}

DIRAC started taking data in November 1998. Since that time we have collected thousands of $\pi^{+} \pi^{-}$Atomic Pairs. For example, the accumulated Nickel target ${ }^{3}$ statistics of 2000, 2001 and 75\% of 2003 runs with tight cuts gives more than 12000 pairs.

However, the accuracy of the measurement, has been limited by systematic effects

\footnotetext{
${ }^{3}$ DIRAC main target is made of Nickel. The former $94 \mu \mathrm{m}$ thickness target was replaced in September 2001 by a $98 \mu m$ one.
} 

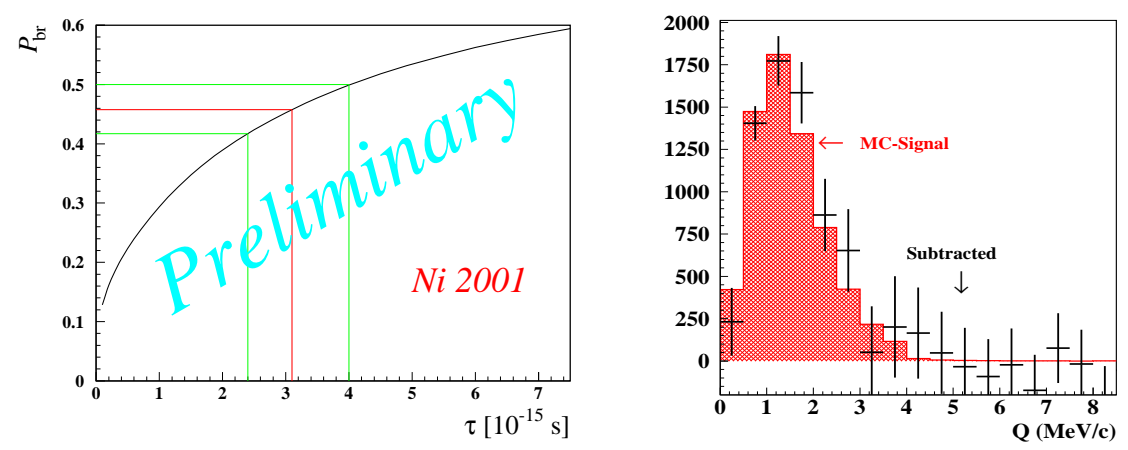

FIGURE 3. Left: Lifetime measurement. Right: Measured spectrum of $\pi^{+} \pi^{-}$Atomic Pairs and comparison to the Monte Carlo corresponding distribution.

as the behavior of the electronic readout of some detectors or the parameterization of Multiple Scattering. In the last two years the major thrust of our research has been the study of systematics, the development of the Monte Carlo simulation of the experiment as well as dedicated measurements like the use of a multi-layer target [9] to deep in the knowledge of our data. These studies are foreseen to be finished early 2004. At the moment we have only a rough estimate of the systematic error of lifetime $\sim 1 \cdot 10^{-15} \mathrm{~s}$. Accounting for that, the result obtained with the use of Nickel target 2001 statistics, the best understood data, gives:

$$
\tau=\left(3.1_{-0.7}^{+0.9}(\text { stat. }) \pm 1 .(\text { syst })\right) \cdot 10^{-15} s .
$$

The pure Monte Carlo analysis leads to a compatible result of

$$
\tau=(2.6 \pm 0.5(\text { stat. }) \pm 1 .(\text { syst })) \cdot 10^{-15} \text { s. }
$$

In figure 3 we can also see the experimental shape of the spectrum of pairs from atomic break-up as defined in equation (7).

\section{REFERENCES}

1. B. Adeva et al. 1995 CERN/SPSLC 95-1 (Geneva: CERN); http: / / www . cern . ch/DIRAC

2. J. Gasser et al., Phys. Rev. D 64016008 (2001)

3. G. Colangelo et al., Phys.Lett. B 488, 261 (2000)

4. C. Santamarina et al., J. Phys. B in press. http://arxiv.org/abs/physics/0306161

5. L.L. Nemenov, Sov. J. Nucl. Phys. 41, 629 (1985)

6. C. Schuetz et al. (DIRAC Collaboration), proceedings of the XXVIIIth Rencontres de Moriond on QCD and Hadronic interactions. http://arxiv.org/abs/hep-ph/0305121

7. DIRAC collaboration: Nucl. Inst. and Meth. A in press. http://www.arxiv.org/abs/hep-ex/0305022

8. L. Afanasyev et al., Nucl. Instr. Meth. A 491, 376 (2002)

9. D. Goldin et al. (DIRAC Collaboration), proceedings of the 8th Conference on the Intersections of Particle and Nuclear Physics. http: //dirac.web.cern.ch/DIRAC/CIPANP2003.pdf 